Original Article

\title{
Effects of dynamic exercise utilizing PNF patterns on the balance of healthy adults
}

\author{
Wontae Gong, PhD, $\mathrm{PT}^{1)}$ \\ 1) Department of Physical Therapy, Korea Nazarene University: 48 Wolbong Ro, Seobuk-gu, Cheonan-Si, \\ Chungcheongnam-do 31172, Republic of Korea
}

\begin{abstract}
Purpose] The purpose of this study is to examine the effects of dynamic exercise utilizing the proprioceptive neuromuscular facilitation patterns accompanied by the abdomen drawing-in on balance of healthy adults. [Participants and Methods] The total number of participants was 30, and 15 were randomly placed in the training group (TG) and 15 in the control group (CG). The participants in the TG have conducted the 3 sets to 5 sets of dynamic exercise utilizing the proprioceptive neuromuscular facilitation patterns per day, 3 times a week for 6 weeks. The balance was measured by the Terax, a balance-measuring device with force plates. [Results] Comparing the TG's balance pre-test and post-test, there was a statistical significance in stability score (SS), weight distribution index (WDI), CD force plates and AC force plates (A: left rearfoot, B: left forefoot, C: right rearfoot, D: right forefoot). [Conclusion] Dynamic trunk stabilization exercise utilizing the proprioceptive neuromuscular facilitation patterns seem to help increase the balance of healthy adults.

Key words: Dynamic exercise, PNF patterns, Balance
\end{abstract}

(This article was submitted Feb. 19, 2019, and was accepted May 5, 2019)

\section{INTRODUCTION}

Balance can be generally divided into static balance and dynamic balance. Static balance refers to the ability to maintain balance while maintaining a posture to prevent the body from moving by placing the center of gravity within the supporting base. Conversely, dynamic balance refers to the ability to maintain balance while the body moves, that is, to maintain a desired posture by placing the center of gravity within the supporting base while the body is moving ${ }^{1)}$. The main trunk muscles that play an important role in maintaining balance are categorized into two types of muscles: 1) the erector spinae and rectus abdominis are the muscles involved in movement while transmitting loads back and forth between the backbone and the pelvis, and 2) the transversus abdominis and multifidus are the local stabilization muscles that maintain the movement of the backbone and posture when a weight load is placed on the spinal structure ${ }^{2}$.

Balance is considered one of the key risk factors for fall, especially in older adults and those with postural control dysfunction $^{3)}$. It is the capability of maintaining the body's center of gravity within the base of support while one maintains a static position, makes a voluntary movement, or reacts to external disruptions. It is a complex construct incorporating multiple biomechanical, neurological, and sensory systems ${ }^{4}$.

Neuromuscular training has been considered an elemental therapeutic strategy to enhance the neurophysiological entity of joints for coordinated functioning ${ }^{5)}$, and an imbalance is associated with a poor joint sense ${ }^{6}$ ). Dynamic exercise utilizing the proprioceptive neuromuscular facilitation (PNF) patterns is an effective exercise method for the development of the multifidus, internal obliquus abdominis, and transversus abdominis muscles, which play an important role in maintaining balance $^{7}$. In addition, PNF pattern exercise can activate joint sense by moving almost all joints of the body under a gravity load condition.

Corresponding author. Wontae Gong (E-mail: owntae@hanmail.net)

(C)2020 The Society of Physical Therapy Science. Published by IPEC Inc.

(c) (i) $(-)$ This is an open-access article distributed under the terms of the Creative Commons Attribution Non-Commercial No DerivaCC ${ }_{\text {BY NC ND }}$ tives (by-nc-nd) License. (CC-BY-NC-ND 4.0: https://creativecommons.org/licenses/by-nc-nd/4.0/) 
Several studies have been conducted on balance, such the effect of core stability, dual tasking, and sensory strategies programs on balance ${ }^{6}$; the effect of backward walking training on balance performance ${ }^{3)}$; the effect of PNF on the balance of older adults with chronic stroke ${ }^{8)}$; and the effect of aquatic exercise on balance ${ }^{9}$. Moreover, studies have analyzed the effect of PNF pattern exercise on the changes in the thickness of the abdominis ${ }^{7)}$ and on postural changes ${ }^{10)}$. However, no study has yet analyzed the effects of dynamic exercise utilizing the PNF patterns on the balance of healthy adult participants. Therefore, this study aims to investigate the effects of dynamic exercise using the PNF patterns, which can activate trunk muscles and joint sense, on the balance of healthy adults.

\section{PARTICIPANTS AND METHODS}

The participants were 30 students attending N University in South Korea. The selection criteria for the participants were as follows: inclusion criteria of normal healthy adults and exclusion criteria of those with a structural abnormality in the vertebrae before participating in this study, those with vertebral pain such as low back pain, those who take medications, those with neurological diseases, those who consumed alcohol before the study, those who were overweight, and those who exercise regularly. The participants were randomized into the training group ( $\mathrm{TG}, \mathrm{M}=2, \mathrm{~F}=13$ ), which was required to participate in the dynamic exercise, and the control group $(\mathrm{CG}, \mathrm{M}=2, \mathrm{~F}=13)$, which was not required to participate in the exercise. The mean age, height, and body weight of the TG group were $22.4 \pm 1.3$ (mean $\pm \mathrm{SD}$ ), $161.1 \pm 1.6 \mathrm{~cm}$, and $56.5 \pm$ $2.6 \mathrm{~kg}$, respectively. The mean age, height, and body weight of the CG group were $21.5 \pm 3.2$ years, $162.7 \pm 2.1 \mathrm{~cm}$, and 57.2 $\pm 2.6 \mathrm{~kg}$, respectively. The $\chi^{2}$ test was used to analyze sex and the independent sample t-test to analyze age, height, and body weight. No statistically significant difference was found in the above analyses $(p>0.05)$, indicating no homogeneity issue between the two groups. This study was approved by the Korea Nazarene University's Institutional Review Board, and the participants were safely protected during all of the processes of the experiment. All of the participants understood the purpose of this study and provided written informed consent prior to their participation in the study in accordance with the ethical standards of the Declaration of Helsinki.

Exercise was performed in standing with limbs moving through 4 PNF patterns of extremities: (1) Lower extremity D1 flexion pattern (LED1flex) is in hip flexion, adduction, and external rotation, knee flexion, and ankle dorsiflexion; (2) Lower extremity D1 extension pattern (LED1ext) is in hip extension, abduction, and internal rotation, knee extension, and ankle plantar flexion; (3) Upper extremity D1 flexion pattern (UED1flex) is in shoulder flexion, adduction, external rotation, and elbow flexion; (4) Upper extremity D1 extension pattern (UED1ext) is in shoulder extension, abduction, internal rotation, and elbow extension. One cycle of exercise was considered 1 time/repetition. One time/repletion of exercise is shown from picture 1 through 10 in Fig. $1^{7)}$. The starting posture was in a small lunge position, with the right leg in LED1 flex, with the left leg in LED1ext, with right arm in UED1ext, and with the left arm in UED1flex. In the starting posture, the entire sole of the right foot was on the floor while the toes/metatarsal heads of the left foot was placed 70 to $80 \mathrm{~cm}$ away from the right foot in the rear right diagonal direction (Picture 1). Once the movement initiates, all 4 limbs moved in the diagonal patterns to get to the mid-point posture (Picture 2 through 4). The mid-point posture was in a single leg stance on the right leg with the right heel raised, with the left leg in LED1flex, with the right arm in UED1flex, and with the left arm in UED1ext (Picture 5). From the mid-point posture to the ending posture, all 4 limbs moved in the diagonal patterns to return to the starting posture (Picture 6 through 10). While exercising, the participants were instructed to keep the trunk in a neutral position, with the trunk and supporting feet steady without movement, and to ensure that the feet always touched the same point in the left and right rear diagonal directions. To select the appropriate exercise intensity and apply the incremental load principle to the participants, in the first and second weeks of the six weeks of exercise, five sets of exercises were performed consisting of two sets of exercises (10 times each for the left and right sides, 20 times in total, was defined as one set), followed by three sets of exercises (20 times each for the left and right sides, 40 times in total, was defined as one set). From the third to sixth weeks, eight sets of exercises were performed consisting of four sets of exercises (10 times each for the left and right sides, 20 times in total, was defined as one set), followed by four sets of exercises (20 times each for the left and right sides, 40 times

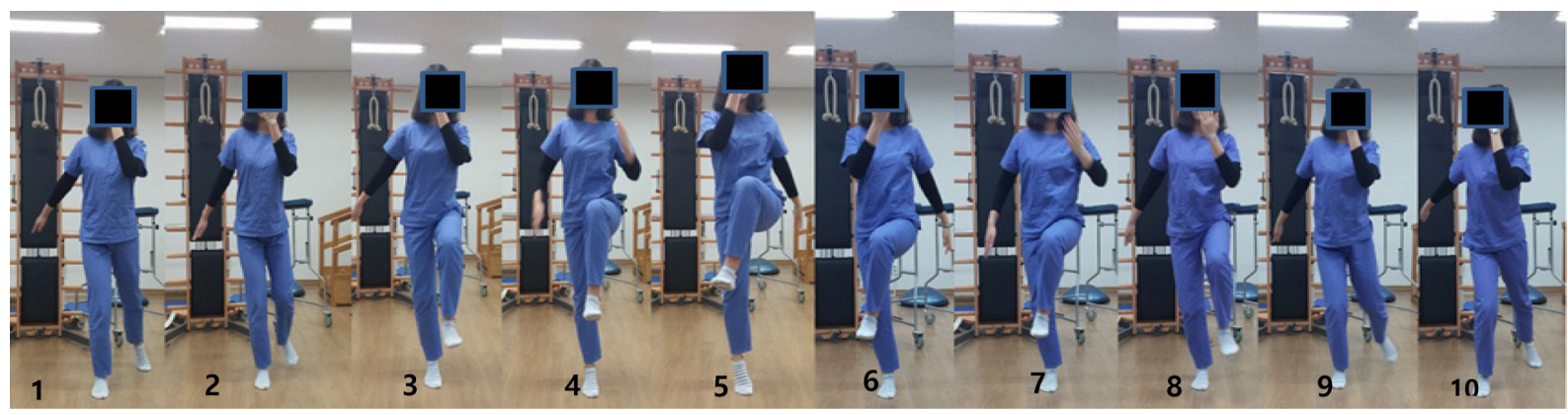

Fig. 1. Dynamic exercise utilizing the PNF patterns. 
in total, was defined as one set). At this time, 30 seconds were given between each set for resting. Including the 5-minute warm-up exercise and the 5-minute cool-down exercise performed before exercise, about 25-30 minutes were spent on the exercise for a session, which was performed three times a week for six weeks. The control group did not engage in any form of regular exercises.

The Tetrax, a balance-measuring device with force plates (Tetrax, Beam Med Ltd., Israel) was used to determine the change in balance before and after the dynamic exercise utilizing the PNF patterns. This instrument was composed of four force plates: two rectangular forefoot plates of $12 \mathrm{~cm}$ in width and $19 \mathrm{~cm}$ in length and two square hindfoot plates of $12 \mathrm{~cm}$ in width and $12 \mathrm{~cm}$ in height. Information on the pressure applied when the participant stood on the plate was amplified, filtered, and then transmitted to a computer for analysis using the Tetrax software program. During the balance evaluation, the participants took off their shoes and placed both feet on the force plates in a quiet room with little external stimuli and maintained the standing posture staring at the front with their eyes open for 32 seconds for the measurement. The stability score (SS) is an index representing the stability of the center of gravity by measuring the variation pattern of the pressure applied to each plate, where larger values indicate greater variation in the center of gravity. The weight distribution index (WDI) represents the percentage of body weight placed on each of the four pressure transducers, where $25 \%$ each of the body weight on one plate is the most ideal form. A value closer to 0 indicates little variation from $25 \%$, whereas a larger value indicates great variation. The synchronization index (SI) represents the result of the comparison of the waveforms of the vibrations measured from two force plates among the four body vibrations measured from each force plate, and a total of six combinations are possible. A negative SI value means a mirror image synchronization of the two waveforms, and a positive SI value means a parallel synchronization of the two waveforms. Whereas negative SI values were observed between the forefoot and the hindfoot of each foot (force plates $\mathrm{AB}, \mathrm{CD}$ ) and between the diagonal plates (force plates $\mathrm{AD}, \mathrm{BC}$ ), positive SI values were observed between the forefeet (force plate $\mathrm{BD}$ ) and the hindfeet (force plate $\mathrm{AC}$ ). The distribution of the values ranges from $-1,000$ to 1,000 . The absolute value for a normal person is usually more than 700 , and the absolute values lower than that indicate poor balance ability ${ }^{11)}$.

The experimental results were statistically analyzed using SPSS 18.0 KO (SPSS, Chicago, IL, USA). After the general characteristics of the participants were determined, paired t-tests were used to analyze pre-test post-test changes within each group, in SS, WDI, AB, CD, AC, and BD. The differences between the two groups were tested using independent t-tests. The statistical significance level, $\alpha$, was set to 0.05 .

\section{RESULTS}

Analysis of pre-test post-test changes in each group, the TG showed statistically significant data for SS, WDI, CD, and $\mathrm{AC}(\mathrm{p}>0.05)$, whereas the CG showed no statistically significant data for all the items ( $>0.05)$ (Table 1$)$. In the comparison of the difference in data values before and after the experiment between the TG and the CG, no items showed statistically significant inter-group differences $(\mathrm{p}>0.05)$, and only the $A B$ item showed a statistically significant difference after the experiment $(\mathrm{p}>0.05)$ (Table 2).

Table 1. Comparison of SS, WDI, AB, CD, AC and BD between pre- and post-test in each group

\begin{tabular}{clcc}
\hline Category & Group & Pre-test & Post-test \\
\hline \multirow{2}{*}{ SS (scores) } & Training G* & $14.7 \pm 4.8$ & $12.0 \pm 2.7$ \\
& Control G & $14.5 \pm 3.2$ & $14.0 \pm 5.1$ \\
WDI (index) & Training G* & $5.5 \pm 2.9$ & $3.6 \pm 1.5$ \\
& Control G & $5.6 \pm 2.3$ & $4.8 \pm 2.3$ \\
AB (index) & Training G & $677.9 \pm 232.9$ & $809.3 \pm 106.2$ \\
& Control G & $668.0 \pm 203.0$ & $681.9 \pm 213.2$ \\
CD (index) & Training G* & $610.6 \pm 187.2$ & $778.1 \pm 131.2$ \\
& Control G & $629.9 \pm 190.8$ & $655.3 \pm 245.9$ \\
AC (index) & Training G* & $551.8 \pm 179.0$ & $671.0 \pm 130.9$ \\
& Control G & $581.0 \pm 163.5$ & $567.8 \pm 192.7$ \\
BD (index) & Training G & $601.4 \pm 256.5$ & $762.5 \pm 181.8$ \\
\hline
\end{tabular}

SS: stability score; WDI: weight distribution index. A: left rearfoot, B: left forefoot, C: right rearfoot, D: right forefoot. Mean \pm SD. ${ }^{*} \mathrm{p}<0.05$. 
Table 2. Comparison of SS, WDI, AB, CD, AC and BD between training group and control group

\begin{tabular}{llcc}
\hline & Category & Training G & Control G \\
\hline \multirow{4}{*}{ Pre-test } & SS (scores) & $14.7 \pm 4.8$ & $14.5 \pm 3.2$ \\
& WDI (index) & $5.5 \pm 2.9$ & $5.6 \pm 2.3$ \\
& AB (index) & $677.9 \pm 232.9$ & $668.0 \pm 203.0$ \\
& CD (index) & $610.6 \pm 187.2$ & $629.9 \pm 190.8$ \\
& AC (index) & $551.8 \pm 179.0$ & $581.0 \pm 163.5$ \\
& BD (index) & $601.4 \pm 256.5$ & $618.6 \pm 191.8$ \\
\cline { 2 - 3 } Post-test & SS (scores) & $12.0 \pm 2.7$ & $14.0 \pm 5.1$ \\
& WDI (index) & $3.6 \pm 1.5$ & $4.8 \pm 2.3$ \\
& AB* (index) & $809.3 \pm 106.2$ & $681.9 \pm 213.2$ \\
Change between & CD (index) & $778.1 \pm 131.2$ & $655.3 \pm 245.9$ \\
pre- and post-test & AC (index) & $671.0 \pm 130.9$ & $567.8 \pm 192.7$ \\
& BD (index) & $762.5 \pm 181.8$ & $629.8 \pm 221.7$ \\
\cline { 2 - 3 } & SS (scores) & $2.7 \pm 5.0$ & $0.4 \pm 7.2$ \\
& WDI (index) & $1.9 \pm 3.0$ & $0.7 \pm 2.9$ \\
& AB (index) & $132.0 \pm 282.8$ & $13.8 \pm 277.0$ \\
& CD (index) & $167.4 \pm 203.3$ & $25.3 \pm 332.1$ \\
& AC (index) & $119.1 \pm 183.4$ & $-13.2 \pm 185.0$ \\
& BD (index) & $161.1 \pm 315.1$ & $11.1 \pm 330.4$ \\
\hline
\end{tabular}

SS: stability score; WDI: weight distribution index. A: left rearfoot, B: left forefoot, C: right rearfoot, D: right forefoot.

Mean \pm SD. ${ }^{*} \mathrm{p}<0.05$.

\section{DISCUSSION}

Balance refers to the ability of the human body to maintain its posture against external forces (i.e., gravity), the ability to maintain the body center on the supporting base within a given environment, and the ability to control limb movement through the effector system, such as muscle strength, range of motion for joints, and flexibility, as a result of the appropriate and effective integration and regulation of afferent information by the central process of the brain center ${ }^{12)}$.

Exercise interventions focused on strength and balance are effective for fall prevention in older people ${ }^{13)}$. Neuromuscular training is responsible for dynamic joint stability, which is defined as training and enhancing unconscious motor responses by stimulating both afferent signals and central mechanisms ${ }^{5)}$.

This study aimed to investigate the effects of dynamic exercise utilizing the PNF patterns on the balance of healthy adults. In a similar study, Nilsagard et al. reported that the application of a core stability, dual tasking, and sensory strategies programs reduced falls and the proportion of fallers and improved balance performance in people with multiple sclerosis, although it did not significantly alter the perceived limitations in walking and balance confidence ${ }^{6}$. Cayco et al. found that proprioceptive neuromuscular facilitation was effective in enhancing balance, strength, and mobility in an older adult with chronic stroke ${ }^{8}$. Gong examined the PNF pattern exercise and found that when the exercise was applied to normal adults for six weeks, the thickness of the external obliquus abdominis, internal obliquus abdominis, and transversus abdominis muscles increased $^{7}$. Cho and Gong showed that the application of PNF pattern exercise to normal adults for six weeks improved trunk inclination, pelvic position, pelvic torsion, pelvic rotation, and the position of their scapular ${ }^{10)}$.

The results of the present study showed that in the comparison between the TG and the CG before and after the experiment, statistical significance was found in SS, WDI, CD, and AC in the TG. This result indicates that the PNF pattern exercise had an effect on balance enhancement, consistent with the study of Cayco et al., which reported that PNF was effective in improving balance, strength, and mobility in older adults with chronic stroke ${ }^{8)}$. These findings suggest that as the stability of the trunk was maintained through the abdomen drawing-in maneuver, the diagonal and spiral movements of the pelvis, lower limbs, scapulae, and upper limbs followed consecutively, leading to the activation of the deep abdominal muscles and the enhanced trunk stability, as the result seems to had a positive influence on balance. The limitations of this study are for healthy people. Future studies will apply PNF patterns to musculoskeletal patients who can maintain their posture. In sum, dynamic exercise using the PNF patterns accompanied by abdomen drawing-in, which can activate the stability of the trunk, is recommended as a way to improve the balance ability of normal adults, except patients who have difficulty in maintaining their posture. 


\section{Funding}

This research was supported by the Korea Nazarene University Research Grants in 2020.

\section{Conflict of interest}

None.

\section{REFERENCES}

1) Wade MG, Jones G: The role of vision and spatial orientation in the maintenance of posture. Phys Ther, 1997, 77: 619-628. [Medline] [CrossRef]

2) Standaert CJ, Weinstein SM, Rumpeltes J: Evidence-informed management of chronic low back pain with lumbar stabilization exercises. Spine J, 2008, 8: 114-120. [Medline] [CrossRef]

3) Wang J, Xu J, An R: Effectiveness of backward walking training on balance performance: a systematic review and meta-analysis. Gait Posture, 2019, 68: 466-475. [Medline] [CrossRef]

4) Heyward VH, Gibson AL: Advanced fitness assessment and exercise prescription, 7th ed. Champaign: Human Kinetics, 2014.

5) Hydar Abbas SA, Karvannan H, Prem V: Effect of neuromuscular training on functional throwing performance and speed in asymptomatic cricket players. J Bodyw Mov Ther, 2019, 23: 502-507. [Medline] [CrossRef]

6) Nilsagård YE, von Koch LK, Nilsson M, et al.: Balance exercise program reduced falls in people with multiple sclerosis: a single-group, pretest-posttest trial. Arch Phys Med Rehabil, 2014, 95: 2428-2434. [Medline] [CrossRef]

7) Gong W: The effects of dynamic exercise utilizing PNF patterns on abdominal muscle thickness in healthy adults. J Phys Ther Sci, 2015, 27: 1933-1936. [Medline] [CrossRef]

8) Cayco CS, Gorgon EJ, Lazaro RT: Effects of proprioceptive neuromuscular facilitation on balance, strength, and mobility of an older adult with chronic stroke: A case report. J Bodyw Mov Ther, 2017, 21: 767-774. [Medline] [CrossRef]

9) Methajarunon P, Eitivipart C, Diver CJ, et al.: Systematic review of published studies on aquatic exercise for balance in patients with multiple sclerosis, Parkinson's disease, and hemiplegia. Hong Kong Physiother J, 2016, 35: 12-20. [Medline] [CrossRef]

10) Cho M, Gong W: The effects of dynamic exercise using the proprioceptive neuromuscular facilitation pattern on posture in healthy adults. J Phys Ther Sci, 2017, 29: 1070-1073. [Medline] [CrossRef]

11) Kohen-Raz R: Application of tetra-ataxiametric posturography in clinical and developmental diagnosis. Percept Mot Skills, 1991, 73: 635-656. [Medline] [CrossRef]

12) Kauffman T: Impact of aging- realted musculoskeletal and ostural changes on fall. Top Geriatr Rehabil, 1990, 5: 34-43. [CrossRef]

13) Martins AC, Santos C, Silva C, et al.: Does modified Otago Exercise Program improves balance in older people? A systematic review. Prev Med Rep, 2018, 11: 231-239. [Medline] [CrossRef] 